\title{
Glycyrrhizin Attenuates Kainic Acid-Induced Neuronal Cell Death in the Mouse Hippocampus
}

\author{
Lidan Luo, Yinchuan Jin, Il-Doo Kim and Ja-Kyeong Lee* \\ Department of Anatomy, Inha University School of Medicine, Incheon 400-712, Korea
}

\begin{abstract}
Glycyrrhizin (GL), a triterpene that is present in the roots and rhizomes of licorice (Glycyrrhiza glabra), has been reported to have anti-inflammatory and anti-viral effects. Recently, we demonstrated that GL produced the neuroprotective effects with the suppression of microglia activation and proinflammatory cytokine induction in the postischemic brain with middle cerebral artery occlusion $(\mathrm{MCAO})$ in rats and improved motor impairment and neurological deficits. In the present study, we investigated whether GL has a beneficial effect in kainic acid (KA)-induced neuronal death model. Intracerebroventricular (i.c.v.) injection of 0.94 nmole $(0.2 \mu \mathrm{g})$ of KA produced typical neuronal death in both CA1 and CA3 regions of the hippocampus. In contrast, administration of $\mathrm{GL}(10 \mathrm{mg} / \mathrm{kg}$, i.p.) $30 \mathrm{~min}$ before KA administration significantly suppressed the neuronal death, and this protective effect was more stronger at $50 \mathrm{mg} / \mathrm{kg}$. Moreover, the GL-mediated neuroprotection was accompanied with the suppression of gliosis and induction of proinflammatory markers (COX-2, iNOS, and TNF- $\alpha$ ). The anti-inflammatory and anti-excitotoxic effects of GL were verified in LPS-treated primary microglial cultures and in NMDA- or KA-treated primary cortical cultures. Together these results suggest that GL confers the neuroprotection through the mechanism of anti-inflammatory and anti-excitotoxic effects in KA-treated brain.
\end{abstract}

Key words: glycyrrhizinic acid, KA, neuroprotection, anti-inflammation

\section{INTRODUCTION}

The administration of kainic acid (KA), an excitatory amino acid L-glutamate analog, is known to induce typical epileptic behavior by mice in a dose-dependent manner $[1,2]$ and to cause neuronal degeneration in limbic structures, such as, the CA1 and CA3 regions of the hippocampus [3-5]. KA-induced hippocampal damage may be triggered directly, but it could also be caused by the hyperactivities of excitatory afferent pathways [2]. Consequently, the progress of neuronal death continues for several

Received May 20,2013, Revised June 9, 2013 ,

Accepted June 10,2013

${ }^{*}$ To whom correspondence should be addressed. TEL: 82-32-890-0913, FAX: 82-32-884-2105 e-mail: jklee@inha.ac.kr days in CA1 and CA3 regions after treatment with KA. Previous studies have also shown that the delayed neuronal cell death occurred in CA1 and CA3 regions of KA-administered mice and is associated with the activations of astrocytes and microglia, with apoptotic neuronal death, and with the enhanced productions of inflammatory cytokines and reactive oxygen species (ROS) [6-8]. Therefore, neuronal cell death detected in mouse hippocampus shows characteristics of both acute and delayed cell death.

Licorice is a natural product that is used to treat liver disease in traditional Chinese medicine. Glycyrrhizin (GL) is extracted from licorice root and has been used in food industry as a flavoring additive. GL has been reported to have a variety of pharmacological effects, in particular, the anti-inflammatory effect of GL and its derivatives has been reported long time ago [9]. Recently, it has been reported that both GL and $18 \beta$-glycyrrhetinic acid (18ßGA, the metabolite of GL) decrease inflammatory
Copyright $\odot$ Experimental Neurobiology 2013. www.enjournal.org
This is an Open Access article distributed under the terms of the Creative Commons Attribution Non-Commercial License (http://creativecommons.org/licenses/by-nc/3.0) which permits unrestricted non-commercial use, distribution, and reproduction in any medium, provided the original work is properly cited. 
response via phosphoinositide-3-kinase/Akt/glycogen synthase kinase-3 $\beta$ (PI3K/Akt/GSK3 $\beta$ ) signaling and glucocorticoid receptor activation, respectively [10]. Furthermore, GL and $18 \beta \mathrm{GA}$ inhibit intracellular ROS production, the inductions of proinflammatory cytokines (TNF- $\alpha$, COX-2, and IL-1 $\beta$ ), and the activations of various transcription factors (NF-kB, PI3K p110 $\delta$, p110 $\gamma$ ) in LPS-treated cells [11]. In addition, GL also inhibits angiogenic activities and the tumor growth in mice, and in the endothelial cells, it decreases the production of reactive oxygen species (ROS) and ERK activation [12].

Recently, accumulating evidences indicate that GL confers neuroprotective effects. Cherng et al. [13] reported that GL has a neuroprotective effect against glutamate-induced excitotoxicity in primary neurons, and Kao et al. [14] reported the neuroprotective effects of GL and 18ßGA in PC12 cells. In addition, it has been shown that GL attenuates rat ischemic spinal cord injury by suppressing inflammatory cytokine induction and HMGB1 secretion [15]. We have also reported that GL efficiently suppressed infarct formation in the postischemic rat brain after middle cerebral artery occlusion (MCAO) and that its neuroprotective effect is accompanied by improvements in motor impairment and neurological deficits, and by the suppressions of microglial activation and proinflammatory cytokine induction [16]. The purpose of this study was to investigate the neuroprotective effects of GL in a KA-induced epileptic animal model. Its anti-epileptic and neuroprotective effects were investigated by examining epileptic behavior and neuronal death, respectively, and the underlying protective mechanisms involved were examined in relation to its anti-inflammatory and anti-excitotoxic effects.

\section{MATERIALS AND METHODS}

\section{Animals}

Male BALB/c mice (25-30 g) were housed under diurnal lighting conditions and allowed food and tap water ad libitum. This study was carried out in strict accordance with the recommendations in the Guide for the Care and Use of Laboratory Animals of the National Institutes of Health. The animal protocol used in this study has been reviewed by the INHA University-Institutional Animal Care and Use Committee (INHA-IACUC) on their ethical procedures and scientific care, and it has been approved (Approval Number INHA-110321-81). Animals were randomly assigned to a KA-treated, KA and GL-treated, or control group. At the start of the experiment, animals weighed 25-30 g and were 10 weeks old.

\section{Kainic acid administration}

Intracerebroventricular (i.c.v.) injection of KA into brain was previously described (Cho et al., 2003). Briefly, male BALB/c mice (25-30 g) were anaesthetized by the intraperitoneal (i.p.) injection of a $2: 1$ mixture $(3.5 \mu \mathrm{l} / \mathrm{g}$ body weight $)$ of ketamine $(50 \mathrm{mg} / \mathrm{ml})$ and xylazine hydrochloride $(23.3 \mathrm{mg} / \mathrm{ml})$, and then placed on a stereotaxic apparatus (Stoelting Co, Wood Dale, IL). Four $\mu \mathrm{l}$ of saline solution containing $\mathrm{KA}(0.2 \mu \mathrm{g})$ was then injected into the right lateral ventricle (stereotaxic coordinates in $\mathrm{mm}$ with reference to the bregma were AP, -2.0; ML, -2.9; DV, -3.7) [17] of mice at $0.5 \mu \mathrm{l} / \mathrm{min}$. After $5 \mathrm{~min}$, the needle was removed over 3 min to minimize backflow. Mice were kept on a warm pad until awake.

\section{Assessment of seizure behavior}

Mice were observed for $150 \mathrm{~min}$ after the KA injection and scored using the following scale (Sperk et al., 1985) [18]: (+1) arrest of motion; $(+2)$ myoclonic jerk of head and neck with brief twitching movements; $(+3)$ unilateral clonic activity, frequent focal convulsions, salivation; $(+4)$ bilateral forelimb tonic and clonic activity, frequent focal convulsions; and $(+5)$ continuous generalized limbic seizures with loss of postural tone, and death within 2 hrs.

\section{Treatment with GL}

GL (Sigma, St. Louis, MO) was dissolved in $0.89 \% \mathrm{NaCl}$ and administered intraperitoneal (i.p.). In total $300 \mu \mathrm{l}$ of GL-containing solution was injected $30 \mathrm{~min}$ before $\mathrm{KA}(0.2 \mu \mathrm{g}$, i.c.v. $)$ treatment.

\section{Sampling of protein}

$\mathrm{BALB} / \mathrm{c}$ mice were sacrificed by cervical dislocation. Then the hippocampus CA1 and CA3 were removed quickly and placed in ice-cold RIPA buffer (50 mM Tris-HCl (pH7.4), 1\% NP-40, $0.25 \%$ sodium-deoxycholate, $150 \mathrm{mM} \mathrm{NaCl}$, and a complete mini protease inhibitor cocktail tablet (Roche, Basel, Switzerland)). After homogenization,lysates were centrifuged at $14,000 \mathrm{rpm}$ at $4^{\circ} \mathrm{C}$ for $15 \mathrm{~min}$ and supernatant liquors were frozen at $-20^{\circ} \mathrm{C}$.

\section{Immunohistochemistry}

Mice brains were fixed with $4 \%$ paraformaldehyde by transcardiac perfusion and post-fixed in the same solution overnight at $4^{\circ} \mathrm{C}$. Fixed brain was incubated with $30 \%$ sucrose overnight and sections $(30 \mu \mathrm{m})$ were prepared by using a vibratome. Immunological staining was performed following standard procedure. Primary antibodies were diluted 1:200 for anti-Neu $\mathrm{N}$ antibody (MAB377, Chemicon, Temecula, CA) and antiionized calcium binding adaptor molecule-1 (Iba-1) (Wako Pure Chemicals, Osaka, Japan), and 1:150 for anti-GFAP antibody (DB Bioscience, San Jose, CA). After washing with PBS containing 0.1\% 
Triton X-100, sections were incubated with anti-mouse IgG (Vector Laboratories, Burlingame, CA) for anti-Neu N and anti-GFAP, or with anti-rabbit IgG (Vector Laboratories, Burlingame, CA) for anti-Iba-1 in PBS for $1 \mathrm{hr}$ at room temperature and visualized using the HRP/3,3'-diaminobenzidine (DAB) system. Numbers of NeuN-positive cells in $0.01 \mathrm{~mm}^{2}\left(0.1 \times 0.1 \mathrm{~mm}^{2}\right)$ for CA1 or in $0.0225 \mathrm{~mm}^{2}\left(0.15 \times 0.15 \mathrm{~mm}^{2}\right)$ for CA3 areas were obtained by counting 12 photographs, 3 photographs per experiment. The representative pictures were presented from three independent experiments.

\section{Immunoblotting}

Fifty $\mu \mathrm{g}$ of proteins were separated by $12 \%$ sodium dodecyl sulfate polyacrylamide gel. After blocking with 5\% non-fat milk for $1 \mathrm{hr}$, membranes were incubated with primary antibodies diluted 1:1,000 for anti-Cox-2 (Santa Cruz Biotechnology, Inc, Delaware, CA), anti-iNOS (Abcam, Cambridge, MA), antiIL-1 $\beta$ (Merck Millipore, Billerica, MA), and anti- $\alpha$-tubulin (Cell Signaling, Denvers, MA) overnight at $4^{\circ} \mathrm{C}$. The next day, membranes were detected using a chemiluminescence kit (Roche, Basel, Switzerland) using anti-rabbit HRP-conjugated secondary antibody (1:2,000, Santa Cruz Biotechnology).

\section{Primary microglial culture}

Primary microglial cultures were prepared as previously described [19]. In brief, cells dissociated from the cerebral hemispheres of 2- to 13-day-old postnatal rat brains Sprague-Dawley strain) were seeded at a density of $1.2 \times 10^{6}$ cells $/ \mathrm{ml}$ in Dulbeccos modified Eagle's medium (DMEM; Gibco, Carlsbad, CA) containing 10\% FBS (Hyclone, Logan, UT) and 1\% penicillin-streptomycin (Gibco, Carsbad, CA). After 2 weeks, microglia were then detached from the flask by mild shaking and filtered through a nylon mesh to remove astrocytes. After centrifugation $(1,000 \times \mathrm{g})$ for $5 \mathrm{~min}$, the cells were resuspended in a fresh DMEM supplemented with $5 \%$ FBS, and plated at a final density of $1 \times 10^{5}$ cells/well on a 24 multi-well culture plate. On the following day, cells were subjected to various treatments. BV2 cells were grown in DMEM supplemented with $1 \%$ penicillin, streptomycin and 5\% FBS.

\section{NO measurements}

Primary microglial cultures plated on 24 -well plates $\left(1 \times 10^{5}\right.$ cells/ well) were treated with lipopolysaccharide (LPS; $200 \mathrm{ng} / \mathrm{ml}$ ) for 24 hrs. To measure the amount of NO produced by microglia, $100 \mu \mathrm{l}$ of the conditioned medium was mixed with an equal volume of Griess reagent (0.5\% sulfanilamide and 0.05\% N-1naphthylethylenediamine), and incubated for $10 \mathrm{~min}$ at room temperature. Absorbances were measured at $550 \mathrm{~nm}$ using a microplate reader.

\section{Primary cortical culture}

Primary cortical cultures, including astrocytes and neurons, were prepared from embryonic day 15.5 mouse cortices and cultured as described previously by Kim et al. [20]. Dissociated cortical cells were plated at a density of approximately $4 \times 10^{5}$ cells per well (five hemispheres per 24-well poly(d-lysine)- and laminincoated plate). Cultures were maintained without antibiotics in MEM containing 5\% horse serum, 5\% fetal bovine serum, $2 \mathrm{mM}$ glutamine, and $21 \mathrm{mM}$ glucose. At day 7 in vitro (DIV 7), when astrocytes had reached confluence underneath neurons, cytosine arabinofuranoside was added to a final concentration of $10 \mu \mathrm{M}$, and cultures were maintained for 2 days to halt microglial growth. Fetal bovine serum and glutamine were not supplemented from day 7 , and media were changed every other day after day 7 . Cultures were used at DIV 12 to 14.

\section{NMDA, KA, or glutamate treatment}

Primary cortical cells were treated with serum-free MEM or HEPES controlled salt solution (HCSS) containing $50 \mu \mathrm{M}$ NMDA (Sigma, St. Louis, MO) for $10 \mathrm{~min}, 100 \mu \mathrm{M}$ KA (Sigma, St. Louis, MO) for 12 hrs, or $100 \mu \mathrm{M}$ glutamate (Sigma, St. Louis, MO) for 1 hr. The medium was then removed and replaced with fresh MEM, and cells were cultured for $24 \mathrm{hrs}$.

\section{LDH assay}

Twenty four hrs after treating cells with NMDA (50 $\mu \mathrm{M}, 10 \mathrm{~min})$, KA $(100 \mu \mathrm{M}, 12 \mathrm{hrs})$, or glutamate $(100 \mu \mathrm{M}, 1 \mathrm{hr}) 50 \mu \mathrm{l}$ aliquots of media and $50 \mu \mathrm{l}$ of $\mathrm{LDH}$ assay reagent (Roche, Mannheim, Germany) were mixed in a 96-well plate and incubated for $15 \mathrm{~min}$. Optical densities were measured using a 96-well plate reader at $490 \mathrm{~nm}$.

\section{Statistical analysis}

Statistical analysis was performed by analysis of variance (ANOVA) followed by the Newman-Keuls test. All data are presented as means \pm SEMs and statistical difference was accepted at the $5 \%$ level.

\section{RESULTS}

\section{GL treatment attenuated kainic acid (KA)-induced neuro- nal death in the hippocampus}

KA (0.2 $\mu$ g, i.c.v.)-injected mice exhibited characteristic epileptic behavior as early as $5 \mathrm{~min}$ after treatment. Seizure progression from 0 to $150 \mathrm{~min}$ after KA administration was scored using 
Sperk's seizure scale [18]. These fixed epileptic behaviors intensified rapidly and the highest level of reaction was observed $90 \mathrm{~min}$ after KA administration (Fig. 1). To examine the neuroprotective effect of GL, it was administered at $30 \mathrm{~min}$ prior to KA (10 or $50 \mathrm{mg} /$ $\mathrm{kg}$, i.p.) and seizure behavior was assessed at the same time points as in KA-administered control animals. Although the severity and duration of seizure behavior were generally lower in the GLadministered group than in KA-administered controls, differences were not statistically significant (Fig. 1).

It has been known that KA administration leads to neuronal death in CA1 and CA3 regions of the hippocampus. To determine degrees of neuronal death, brain tissue slices containing hippocampus were stained with anti-NeuN antibody at 2 days after KA treatment. In saline-treated control animals, mean numbers of NeuN ${ }^{+}$cells in the CA1 $\left(0.1 \times 0.1 \mathrm{~mm}^{2}\right)$ and CA3

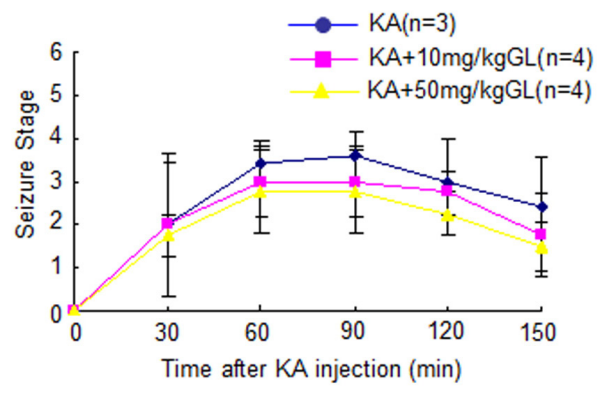

Fig. 1. No change in KA-induced epileptic behavior by GL. GL (10 or $50 \mathrm{mg} / \mathrm{kg}$ ) was administered intraperitoneally (i.p.) $30 \mathrm{~min}$ prior to KA injection $(0.2 \mu$ g, i.c.v. $)$. The seizure activity was scored using the rating scale devised by Sperk et al. [18]. Temporal seizure activities are presented as means \pm SEMs $(\mathrm{n}=8)$. $\left(0.15 \times 0.15 \mathrm{~mm}^{2}\right)$ regions (indicated as black box in Fig. $\left.2 \mathrm{~A}\right)$ of the hippocampus were $99.3 \pm 18.7(n=12)$ and $178.7 \pm 9.1(n=12)$, respectively (Fig. 2A, E, F) and in the KA-administered controls, mean numbers of NeuN+ cells were $42.9 \pm 6.9(n=12)$ and $51.7 \pm 9.1$ $(\mathrm{n}=12)$, respectively (Fig. 2B, E, F). When $10 \mathrm{mg} / \mathrm{kg}$ of GL was administered $30 \mathrm{~min}$ prior to KA, KA-induced neuronal death was significantly suppressed, and mean numbers of NeuN+ cells were 53.5 $\pm 9.4(n=12)$ and 97.2 $\pm 9.7(n=12)$, respectively (Fig. 2C, D, F). KA-induced neuronal death was further suppressed by the administration of $50 \mathrm{mg} / \mathrm{kg}$ of GL and corresponding mean numbers of $\mathrm{NeuN}^{+}$cells were $63.0 \pm 8.9(\mathrm{n}=12)$ and $140.8 \pm 13.5$ $(\mathrm{n}=12)$ (Fig. 2D, E, F). When GL (50 mg/kg) was administered 30 min after KA injection, KA-induced neuronal death was also significantly suppressed although the effects appeared to be slightly weaker than that observed in 30 min pre-administered animals (data not shown). These results indicated that GL suppressed KAinduced neuronal death in the mouse hippocampus.

\section{GL suppressed KA-induced astrocyte and microglia activa- tions in CA1 and CA3}

We examined whether GL affected inflammatory processes in KA-injected mice. Anti-GFAP and anti-Iba- 1 antibodies were used to access astrocyte and microglial activations, respectively. In saline-treated control animals, immunostaining with antiGFAP antibody revealed that astrocyte cell bodies were small and their processes were long and thin (Fig. 3A). However, at 2 days after KA injection, astrocyte cell bodies were enlarged and their processes were shorter and thicker, that is, they possessed the characteristics of activating astrocytes (Fig. 3B). At 4 days after KA administration, astrocytes were activated further and exhibited the

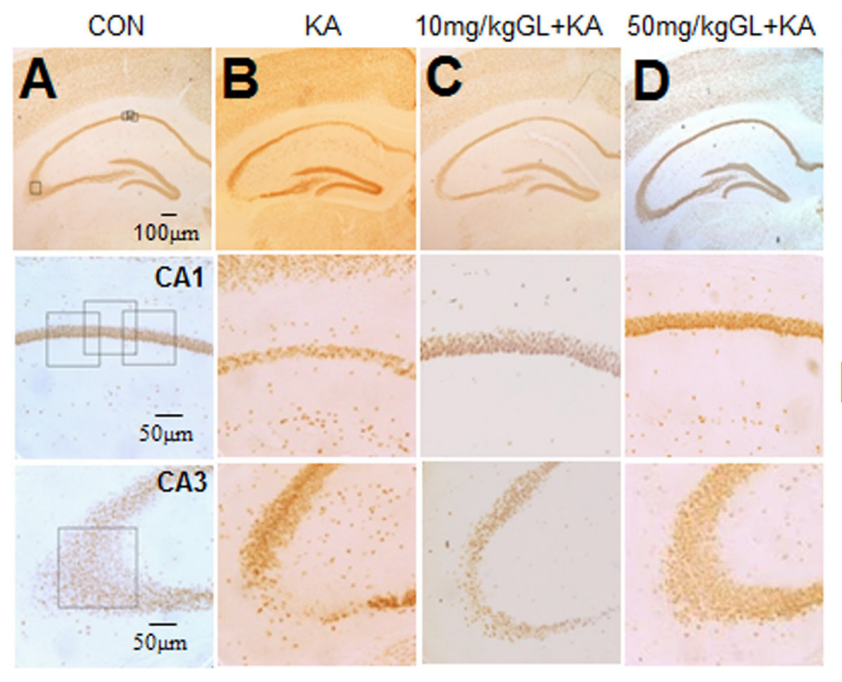

E CA1

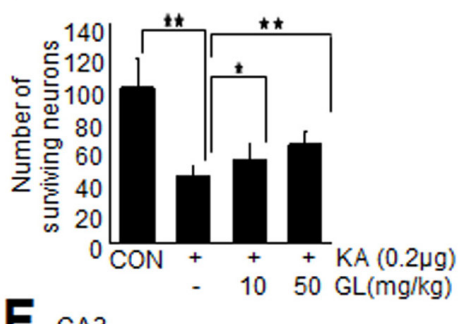

F ${ }_{\text {CA3 }}$

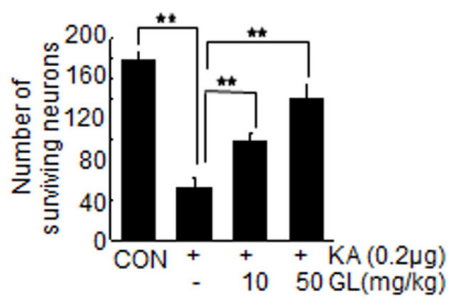

Fig. 2. Attenuation of kainic acidinduced neuronal death in the hippocampus by GL. GL ( 10 or 50 $\mathrm{mg} / \mathrm{kg}$ ) was administered intraperitoneally (i.p.) $30 \mathrm{~min}$ prior to KA $(0.2 \mu$ g, i.c.v. $)$. Hippocampal sections were prepared 2 days after KA administration and stained with anti-NeuN ${ }^{+}$antibody. Numbers of $\mathrm{NeuN}^{+}$cells were counted in the CA1 $\left(0.01 \mathrm{~mm}^{2}, 0.1 \times 0.1 \mathrm{~mm}\right)$ and CA3 $\left(0.0225 \mathrm{~mm}^{2}, 0.15 \times 0.15\right.$ $\mathrm{mm}$ ) regions of the hippocampus (indicated as black boxes) and presented as means \pm SEMs. ${ }^{*} \mathrm{p}<0.05$ and ${ }^{* *} \mathrm{p}<0.01$. Scale bars represent $100 \mu \mathrm{m}$ in the upper panels of A-D and $50 \mu \mathrm{m}$ in the middle and lower panels of A-D. 

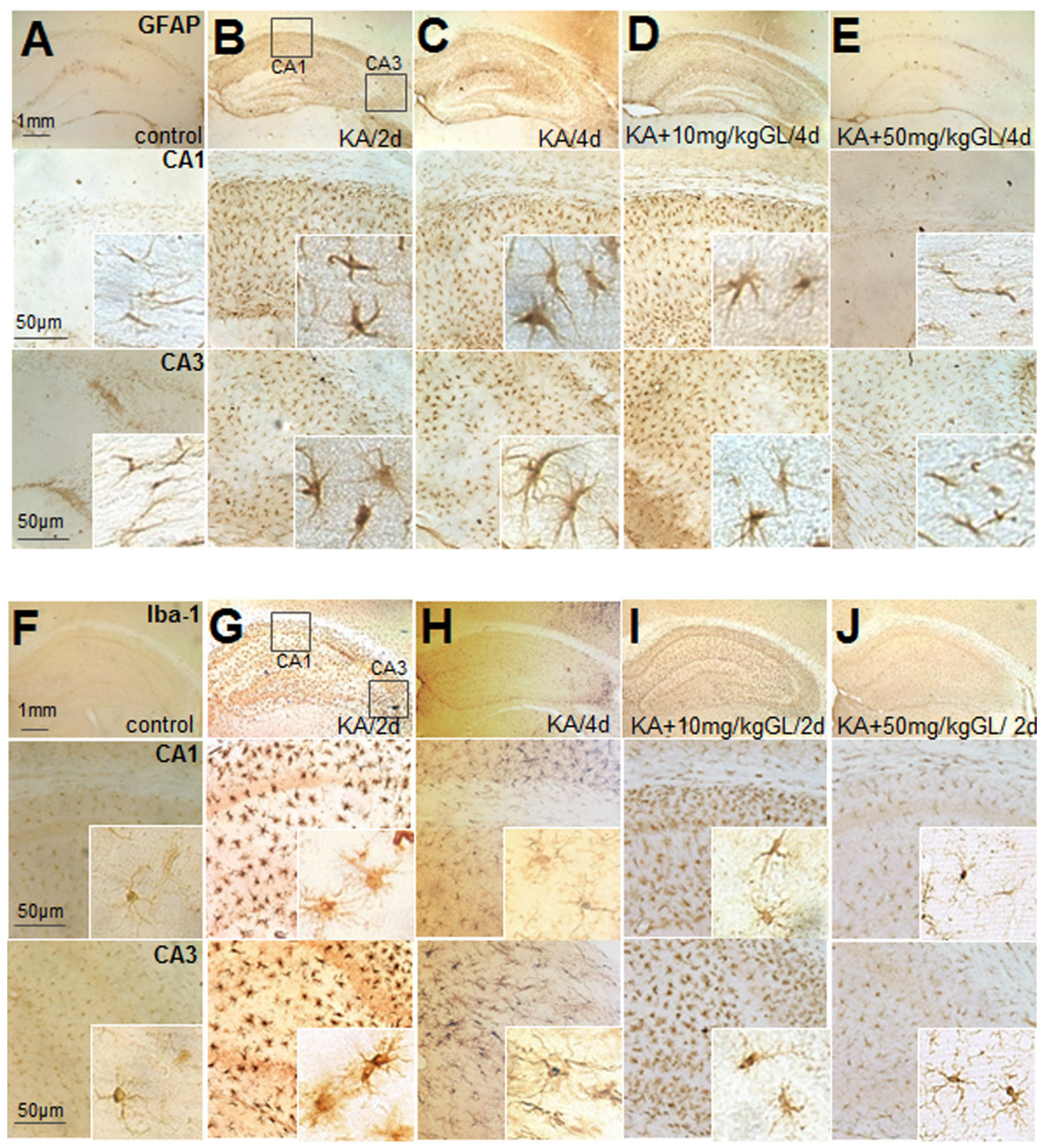

Fig. 3. Suppression of KA-induced astrocyte and microglia activations by GL. GL was administered (10 or $50 \mathrm{mg} / \mathrm{kg}$, i.p.) $30 \mathrm{~min}$ prior to KA treatment $(0.2 \mu$ g, i.c.v. $)$. Hippocampal sections were prepared at 2 and 4 days post-KA treatment $(0.2 \mu$ g, i.c.v. $)$ and stained with anti-GFAP (A-E) or anti-Iba-1 (F-J) antibody. Photographs in middle and lower panels are high magnification micrographs of the $\mathrm{CA} 1$ and $\mathrm{CA} 3$ regions indicated as black boxes in B and G. The insets in each picture are high magnification micrographs of CA1 and $\mathrm{CA} 3$ regions. Representative picture from three independent experiments were presented. Scale bars represent $4 \mathrm{~mm}$ in the upper panels and $50 \mu \mathrm{m}$ in the middle and lower panels. characteristic star-shape of activated astrocytes (Fig. 3C). However, when $10 \mathrm{mg} / \mathrm{kg}$ GL was injected $30 \mathrm{~min}$ prior to KA, astrocyte activation at 4 days after KA administration was remarkably suppressed (Fig. 3D) and it was further suppressed by $50 \mathrm{mg} / \mathrm{kg}$ GL, adopting morphology of the resting state (Fig. 3E). Staining with anti-Iba-1 antibody (a marker of cells of myeloid origin [21]) showed that in saline-treated control animals, Iba-1 -positive cells in the hippocampus were ramified (Fig. 3F). However, 2 days after KA injection, cell bodies of Iba-1-positive cells were enlarged and cytoplasmic processes were thicker and shorter in CA1 and CA3, displaying activated morphology of microglia (Fig. 3G). In contrast to astrocyte activation, staining with anti-Iba-1 antibody detected fewer number of Iba-1-positive cells exhibiting activated morphology at 4 days after KA administration (Fig. 3H). In 10 $\mathrm{mg} / \mathrm{kg}$ GL-administered animals, number of Iba-1-positive cells exhibiting activated morphology was significantly suppressed at
2 days post-KA (Fig. 3I) and it was almost completely suppressed by $50 \mathrm{mg} / \mathrm{kg} \mathrm{GL}$, wherein cell bodies of Iba-1-positive cells were thin and their cytoplasmic processes were ramified (Fig. 3J). These results suggest that the activations of microglia/macrophage and astrocytes in the hippocampus of KA-administered animals were suppressed by GL.

\section{GL suppressed KA-induced proinflammatory marker production}

Next, we examined whether the inhibition of astrocyte and microglia activation by GL is accompanied by the suppression of proinflammatory marker production in the hippocampus. Levels of proinflammatory markers in KA-injected animals were assessed by immunoblotting with anti-COX-2, anti-iNOS, and anti-IL-1 $\beta$ antibodies. Protein samples were prepared from both CA1 and CA3 hippocampal regions (indicated in Fig. 4A) one day 

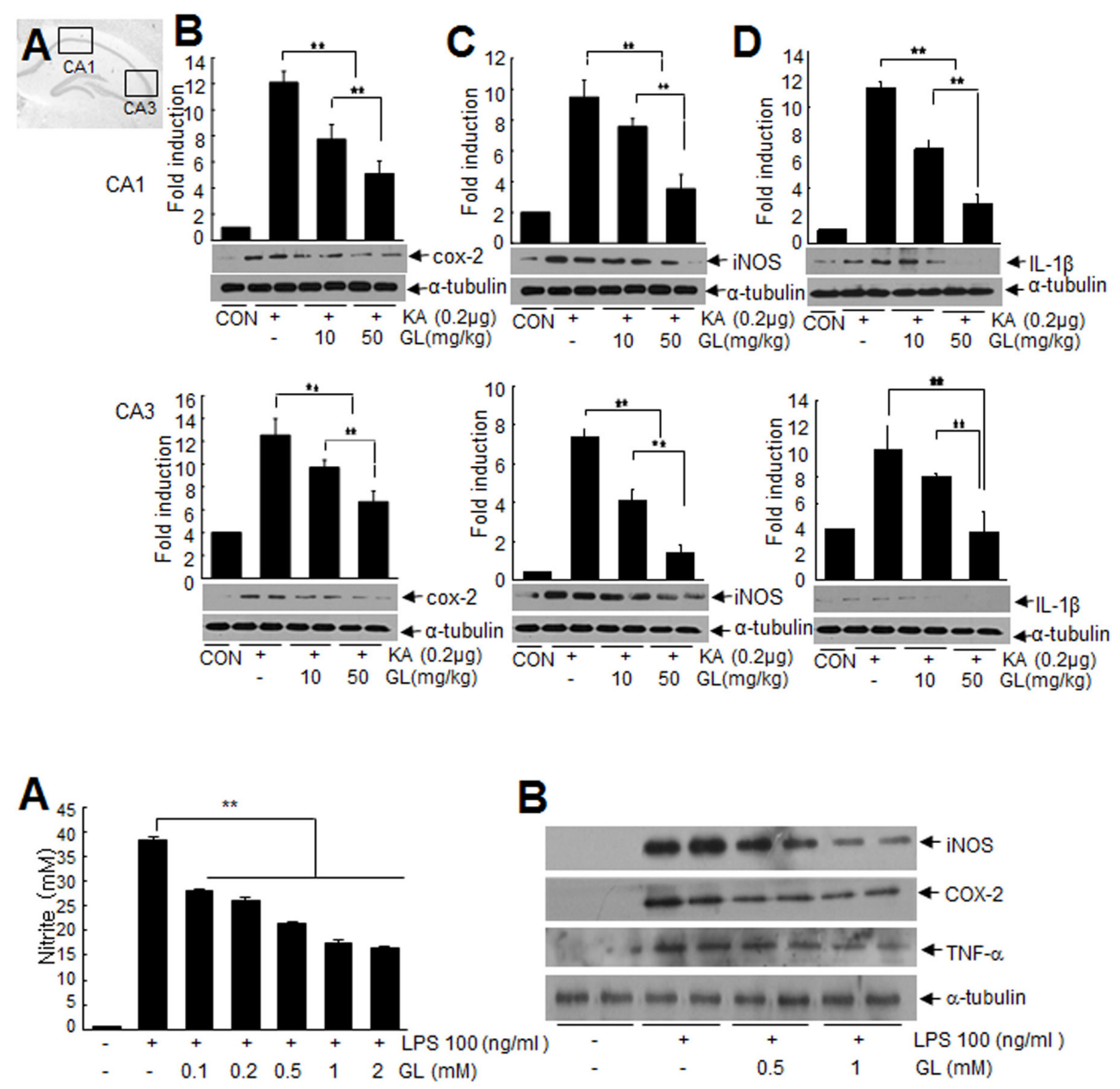

B

C

iNOS

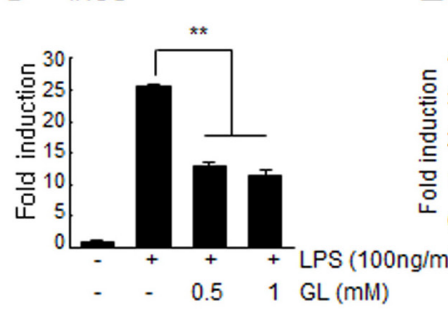

D cox-2
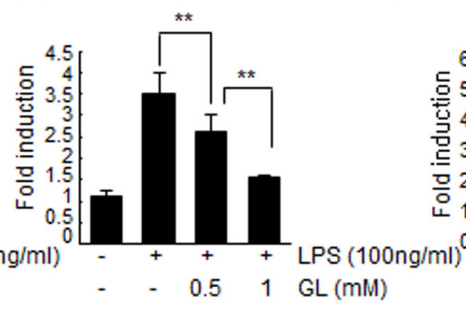

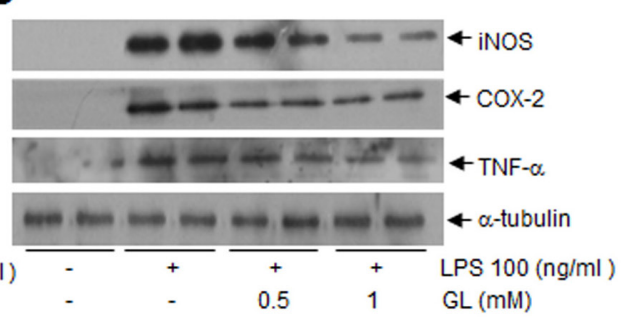

$\mathbf{E}_{\mathrm{TNF}-\alpha}$

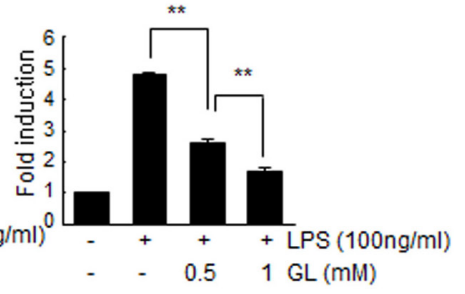

Fig. 4. Suppression of KA-induced proinflammatory marker production by GL. GL (10 or 50 $\mathrm{mg} / \mathrm{kg}$, i.p.) was administered 30 min prior to KA $(0.2 \mu$ g, i.c.v. $)$ and levels of proinflammatory markers were assessed by immunoblotting. Protein samples were prepared at 1 day post-KA administration from the $\mathrm{CA} 1$ and $\mathrm{CA} 3$ regions (indicated in $\mathrm{A}$ ) and results are presented as means \pm SEMs $(n=4)$ ${ }^{* *} \mathrm{p}<0.01$. after KA injection. COX-2 expression was notably increased both in CA1 and CA3 (Fig. 4B). After treatment with $10 \mathrm{mg} / \mathrm{kg}$ of GL, COX-2 expression was significantly suppressed and it was further suppressed by $50 \mathrm{mg} / \mathrm{kg}$ GL (Fig. 4B). iNOS and IL- $1 \beta$ expressions were also notably increased in KA-injected hippocampi and suppressed dose-dependently by GL (Fig. 4C, D). These results indicate that KA-induced proinflammatory marker productions were suppressed by GL.

\section{GL suppressed the LPS-induced activation of microglial cells}

To investigate the anti-inflammatory effect of GL, the GL- dependent suppression of microglial activation was examined in primary microglial cultures. Cells were stimulated with LPS (0.1 $\mu \mathrm{g} / \mathrm{ml}$ ) for $24 \mathrm{hrs}$ with and without GL co-treatment, and nitrite production was measured. GL co-treatment reduced LPS-induced nitrite production dose-dependently; maximum inhibition was achieved at a GL concentration of $1 \mathrm{mM}$ (Fig. 5A). In addition, GL dose-dependently repressed the inductions of proinflammatory markers (Cox-2, TNF- $\alpha$, and iNOS) in LPS-treated primary microglial cultures (Fig. 5B-E). In contrast, $\alpha$-tubulin levels were not changed at any GL concentration tested (Fig. 5B). These results indicate that GL suppresses LPS-induced microglial activation. 


\section{Anti-excitotoxic effects of GL in primary cortical cultures}

GL-mediated suppression of neuronal death in KA-administered animal prompted us to examine an anti-excitotoxic effect of GL in primary cortical cultures. Since glutamate-mediated excitotoxic cell death is the main neurotoxic mechanism in epilepsy [22], NMDA, glutamate, and KA were used to induce excitotoxic damage in primary cortical cultures and to examine the antiexcitotoxic effects of GL. Severe neuronal damage was observed 2 hrs after NMDA treatment $(50 \mu \mathrm{M}, 10 \mathrm{~min})$ in primary cortical cultures (Fig. 6A). However, co-treatment with $0.5 \mathrm{mM}$ of GL significantly reduced $\mathrm{LDH}$ release, and treatment with $1 \mathrm{mM}$ of GL further reduced LDH release (Fig. 6A). Similar neuroprotective effects were also observed in KA $(500 \mu \mathrm{M}, 12$ hrs)- or glutamate ( $100 \mu \mathrm{M}, 1 \mathrm{hr}$ )-treated primary cortical cultures (Figs. 6B, C), although, the protective efficacy of GL differed for the different treatments (Fig. 6). Together these results indicate that antiexcitotoxic effects in neurons contributes to the neuroprotective effects of GL in the postischemic brain.
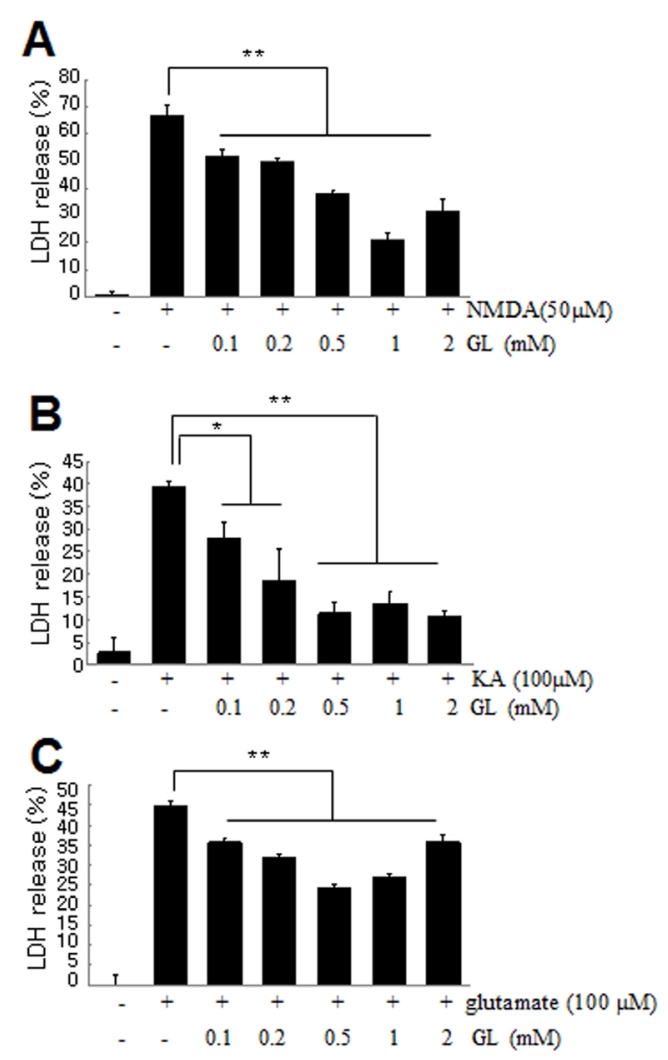

Fig. 6. Effects of GL in NMDA-, KA, or glutamate-treated primary cortical cultures. LDH levels in primary cortical cultures were measured at $24 \mathrm{hrs}$ after NMDA ( $50 \mu \mathrm{M}, 10 \mathrm{~min}$ (A) or KA (150 $\mu \mathrm{M}, 12 \mathrm{hrs})$ (B), or glutamate $(100 \mu \mathrm{M}, 1 \mathrm{hr})$ treatment in the presence or absence of GL ( 0.1 , $0.2,0.5,1$, or $2 \mathrm{mM})$. LDH levels are presented as means \pm SEMs. $(n=4)$. ${ }^{*} \mathrm{p}<0.05,{ }^{* *} \mathrm{p}<0.01$.

\section{DISCUSSION}

This study shows that GL has a strong neuroprotective effect in KA-administered mice. GL treatment was found to decrease neuronal death in the hippocampus, inhibited astrocyte and microglia activation in KA-administered mouse brain, and suppressed inflammatory marker induction. In addition, GL significantly suppressed NMDA-, KA-, or glutamate-induced neuronal death, indicating that GL confers anti-excitotoxic effect. In our previous studies, we have described inhibitions of microglia activation and concomitant inflammatory cytokine inductions by GL in the postischemic brain [16]. The present result, together with our previous report, suggests that neuroprotective effect of GL is, at least in part, attributable to its anti-inflammatory and anti-excitotoxic functions.

Recently, a number of studies have indicated that inflammation might be a consequence and also a cause of epilepsy [23]. It has been reported that inflammation contributes substantially to delayed brain damage after acute injury and that it detrimentally affects neurological outcome following KA-induced epileptic seizures $[6,8]$. In particular, astrocytes and microglia are activated and first upregulate proinflammatory cytokines (IL-1 $\beta$, TNF, and IL-6) and cytokine receptor [24] and subsequently, an ensuing inflammatory process is induced not only in astrocyte and microglia but endothelial cells and peripheral immune cells migrated into the brain parenchyma. A numbers of studies have shown anti-inflammatory effects of GL in animal disease models, which is in accord with our findings. For example, GL was found to alleviate inflammatory lung disease, chronic obstructive pulmonary disease (COPD) [10], to reduce LPSinduced acute respiratory distress syndrome (ARDS) lethality [25], and to alleviate ovalbumin-induced allergic asthma in BALB/ c mice [26]. Regarding the molecular mechanism underlying anti-inflammatory effect of GL, blocking the activations of NF$\mathrm{kB}, \mathrm{PI} 3 \mathrm{~K}, \mathrm{p} 110 \delta$, and $\mathrm{p} 110 \gamma$ has been reported [11]. In addition, inhibition of ROS formation and redox-sensitive signaling (nuclear factor-kB (NF- $\mathrm{B}$ ), Jun N-terminal kinase, and p38 mitogen-activated protein kinase) by GL has been shown to be responsible for suppression of pro-inflammatory gene expression [27]. Recently, the suppression of HMGB1 release by GL has also been reported to reduce apoptosis and inflammatory cytokines induction after transient spinal cord I/R injury [28] and in animal model of MCAO [16].

Although GL fails to inhibit proconvulsant effect of KA, we found that the anti-excitotoxic effect of GL might also contribute to its neuroprotective effect in a KA-induced seizure animal model (Fig. 6). It has been reported that in rat hippocampal slices and 
primary neuronal cultures, GL can suppress glutamate-induced neurotoxicity and reduce cell death [13], which corroborates our results. In addition, carbenoxolon, a synthetic derivative of GL, has also been reported to block NMDA receptors and to impair longterm potentiation in mouse hippocampal slices [29]. It is of note that we also confirmed neuroprotective and anti-excitotoxic effects of carbenoxolon in stroke animal model and in KA-induced epilepsy animal model (Luo et al., unpublished data). Although, carbenoxolon has been known to function as a gap junction uncoupler [30], the mechanisms underlying the neuroprotective effects of GL need further study.

In terms of its neuroprotective potency, Hwang et al. [31] reported that the neuroprotective effect of roasted licorice against ischemic damage is greater than raw form and it might be through antioxidant effect. We have reported that SNMC, a glycyrrhizincontaining preparation developed for chronic liver disease, has a marked neuroprotective function in the postischemic brain via its anti-inflammatory effects [32] and SNMC shows significantly higher neuroprotective potency compared to an equivalent dose of pure glycyrrhizin, in terms of reducing infarct volume and improving neurological deficits [32]. Considering robust neuroprotective effects of GL in KA-induced animal seizure model in the present study and in MCAO animal model in our previous reports, investigation on the improvement of therapeutic potency of GL by modification and by producing formulation is worth pursuing.

\section{ACKNOWLEDGEMENTS}

This research was financially supported by Inha University Research Grant for Ja-Kyeong Lee.

\section{REFERENCES}

1. Coyle JT (1983) Neurotoxic action of kainic acid. J Neurochem 41:1-11.

2. Sperk G (1994) Kainic acid seizures in the rat. Prog Neurobiol 42:1-32.

3. Frederickson CJ, Hernandez MD, McGinty JF (1989) Translocation of zinc may contribute to seizure-induced death of neurons. Brain Res 480:317-321.

4. Choi DW (1990) Methods for antagonizing glutamate neurotoxicity. Cerebrovasc Brain Metab Rev 2:105-147.

5. Weiss JH, Sensi SL, Koh JY (2000) Zn(2+): a novel ionic mediator of neural injury in brain disease. Trends Pharmacol Sci 21:395-401.

6. Kim SW, Yu YM, Piao CS, Kim JB, Lee JK (2004) Inhibition of delayed induction of p38 mitogen-activated protein kinase attenuates kainic acid-induced neuronal loss in the hippocampus. Brain Res 1007:188-191.

7. Weise J, Engelhorn T, Dörfler A, Aker S, Bähr M, Hufnagel A (2005) Expression time course and spatial distribution of activated caspase-3 after experimental status epilepticus: contribution of delayed neuronal cell death to seizureinduced neuronal injury. Neurobiol Dis 18:582-590.

8. Penkowa M, Florit S, Giralt M, Quintana A, Molinero A, Carrasco J, Hidalgo J (2005) Metallothionein reduces central nervous system inflammation, neurodegeneration, and cell death following kainic acid-induced epileptic seizures. J Neurosci Res 79:522-534.

9. Finney RS, Somers GF (1958) The antiinflammatory activity of glycyrrhetinic acid and derivatives. J Pharm Pharmacol 10:613-620.

10. Kao TC, Shyu MH, Yen GC (2010) Glycyrrhizic acid and $18 \beta$-glycyrrhetinic acid inhibit inflammation via PI3K/Akt/ GSK3 $\beta$ signaling and glucocorticoid receptor activation. J Agric Food Chem 58:8623-8629.

11. Wang CY, Kao TC, Lo WH, Yen GC (2011) Glycyrrhizic acid and $18 \beta$-glycyrrhetinic acid modulate lipopolysaccharideinduced inflammatory response by suppression of NF- $\kappa B$ through PI3K p $110 \delta$ and p $110 \gamma$ inhibitions. J Agric Food Chem 59:7726-7733.

12. Kim KJ, Choi JS, Kim KW, Jeong JW (2013) The anti-angiogenic activities of glycyrrhizic acid in tumor progression. Phytother Res 27:841-846.

13. Cherng JM, Lin HJ, Hung MS, Lin YR, Chan MH, Lin JC (2006) Inhibition of nuclear factor $\kappa \mathrm{B}$ is associated with neuroprotective effects of glycyrrhizic acid on glutamateinduced excitotoxicity in primary neurons. Eur J Pharmacol 547:10-21.

14. Kao TC, Shyu MH, Yen GC (2009) Neuroprotective effects of glycyrrhizic acid and $18 \beta$-glycyrrhetinic acid in PC12 cells via modulation of the PI3K/Akt pathway. J Agric Food Chem 57:754-761.

15. Gong G, Yuan LB, Hu L, Wu W, Yin L, Hou JL, Liu YH, Zhou LS (2012) Glycyrrhizin attenuates rat ischemic spinal cord injury by suppressing inflammatory cytokines and HMGB1. Acta Pharmacol Sin 33:11-18.

16. Kim SW, Jin Y, Shin JH, Kim ID, Lee HK, Park S, Han PL, Lee JK (2012) Glycyrrhizic acid affords robust neuroprotection in the postischemic brain via anti-inflammatory effect by inhibiting HMGB1 phosphorylation and secretion. Neurobiol Dis 46:147-156.

17. Franklin KB, Paxinos G (1997) The mouse brain in stereotaxic 
coordinates. Academic Press Inc., San Diego, CA.

18. Sperk G, Lassmann H, Baran H, Seitelberger F, Hornykiewicz O (1985) Kainic acid-induced seizures: dose-relationship of behavioural, neurochemical and histopathological changes. Brain Res 338:289-295.

19. Kim JB, Choi JS, Yu YM, Nam K, Piao CS, Kim SW, Lee MH, Han PL, Park JS, Lee JK (2006) HMGB1, a novel cytokinelike mediator linking acute neuronal death and delayed neuroinflammation in the postischemic brain. J Neurosci 26:6413-6421.

20. Kim SW, Lim CM, Kim JB, Shin JH, Lee S, Lee M, Lee JK (2011) Extracellular HMGB1 released by NMDA treatment confers neuronal apoptosis via RAGE-p38 MAPK/ERK signaling pathway. Neurotox Res 20:159-169.

21. Imai Y, Ibata I, Ito D, Ohsawa K, Kohsaka S (1996) A novel gene ibal in the major histocompatibility complex class III region encoding an EF hand protein expressed in a monocytic lineage. Biochem Biophys Res Commun 224:855862.

22. Choi DW, Koh JY, Peters S (1988) Pharmacology of glutamate neurotoxicity in cortical cell culture: attenuation by NMDA antagonists. J Neurosci 8:185-196.

23. Vezzani A, French J, Bartfai T, Baram TZ (2011) The role of inflammation in epilepsy. Nat Rev Neurol 7:31-40.

24. Vezzani A, Granata T (2005) Brain inflammation in epilepsy: experimental and clinical evidence. Epilepsia 46:1724-1743.

25. Yu Z, Ohtaki Y, Kai K, Sasano T, Shimauchi H, Yokochi T, Takada H, Sugawara S, Kumagai K, Endo Y (2005) Critical roles of platelets in lipopolysaccharide-induced lethality: effects of glycyrrhizin and possible strategy for acute respiratory distress syndrome. Int Immunopharmacol 5:571580.
26. Ram A, Mabalirajan U, Das M, Bhattacharya I, Dinda AK, Gangal SV, Ghosh B (2006) Glycyrrhizin alleviates experimental allergic asthma in mice. Int Immunopharmacol 6:1468-1477.

27. Michaelis M, Geiler J, Naczk P, Sithisarn P, Leutz A, Doerr HW, Cinatl J Jr (2011) Glycyrrhizin exerts antioxidative effects in $\mathrm{H} 5 \mathrm{~N} 1$ influenza A virus-infected cells and inhibits virus replication and pro-inflammatory gene expression. PLoS One 6:e19705.

28. Zhai CL, Zhang MQ, Zhang Y, Xu HX, Wang JM, An GP, Wang YY, Li L (2012) Glycyrrhizin protects rat heart against ischemia-reperfusion injury through blockade of HMGB1dependent phospho-JNK/Bax pathway. Acta Pharmacol Sin 33:1477-1487.

29. Chepkova AN, Sergeeva OA, Haas HL (2008) Carbenoxolone impairs LTP and blocks NMDA receptors in murine hippocampus. Neuropharmacology 55:139-147.

30. Davidson JS, Baumgarten IM (1988) Glycyrrhetinic acid derivatives: a novel class of inhibitors of gap-junctional intercellular communication. Structure-activity relationships. J Pharmacol Exp Ther 246:1104-1107.

31. Hwang IK, Lim SS, Choi KH, Yoo KY, Shin HK, Kim EJ, YoonPark JH, Kang TC, Kim YS, Kwon DY, Kim DW, Moon WK, Won MH (2006) Neuroprotective effects of roasted licorice, not raw form, on neuronal injury in gerbil hippocampus after transient forebrain ischemia. Acta Pharmacol Sin 27:959-965.

32. Jin YC, Kim SW, Cheng F, Shin JH, Park JK, Lee S, Lee JE, Han PL, Lee M, Kim KK, Choi H, Lee JK (2011) The effect of biodegradable gelatin microspheres on the neuroprotective effects of high mobility group box $1 \mathrm{~A}$ box in the postischemic brain. Biomaterials 32:899-908. 